\title{
Tooth Reduction Guide Using Silicone Registration Material Along with Vacuum-Formed Thermoplastic Matrix
}

\author{
Won-suk Oh, DDS, MS, Berna Saglik, DDS, MS, \& Kenneth B. May, DDS, MS \\ Division of Prosthodontics, Department of Biologic \& Materials Sciences, University of Michigan School of Dentistry, Ann Arbor, MI
}

\author{
Keywords \\ Tooth reduction guide; silicone registration \\ material; vacuum-formed thermoplastic matrix.

\section{Correspondence} \\ Won-suk Oh, Department of Biologic \& \\ Materials Sciences, University of Michigan \\ School of Dentistry, 1011 N. University, Rm \\ K1014A, Ann Arbor, Ml 48109-1078. \\ E-mail: ohws@umich.edu
}

Accepted December 5, 2008

doi: 10.1111/j.1532-849X.2009.00506.x

\begin{abstract}
Adequate tooth reduction is a prerequisite for function, esthetics, and longevity of fixed restorations. A tooth reduction guide may be useful for establishing the proper angulation of the tooth and maximizing periodontal health and restorative success. This article describes a simple and versatile technique for an accurate evaluation of tooth reduction for fixed restorations by using a color-contrasting positive guide of a silicone occlusal registration material processed inside a vacuum-formed clear thermoplastic matrix.
\end{abstract}

Adequate tooth reduction is an important requirement for function, esthetics, and longevity of fixed restorations, especially metal ceramic and all-ceramic restorations. ${ }^{1}$ Periodontal disease, ${ }^{2}$ increased risk of chipping or fracture of porcelain, ${ }^{3}$ or compromised esthetics ${ }^{4}$ have been associated with overcontoured restorations, insufficient or excessive thickness of the porcelain layer, or disharmonious proportion of opaque and body porcelain layers. Ideal tooth preparation should ensure uniform reduction and sufficient clearance to allow adequate thickness of the final restoration without impairing periodontal health, esthetics, and structural durability. ${ }^{1-4}$

A tooth reduction guide is recommended to help create uniform space for the restoration and prevent an undesirable situation, in particular when the tooth is misaligned, tilted, rotated, or needs significant alterations. ${ }^{5-11}$ As the treatment plan for the fixed restoration is established, an ideal tooth contour is established by means of a diagnostic waxing, including alteration of occlusal vertical dimension and orientation of occlusal plane when needed.

A typical reduction guide is fabricated either of poly vinyl siloxane (PVS) putty impression material ${ }^{6,7,10}$ or thermoplastic sheet. $5,8,9,11$ The guide is seated in place intraorally, and the clearance for the restoration is evaluated visually and quantified. The PVS putty impression material is preferred for intricate prosthodontic procedures because it duplicates and accurately transfers the diagnostic waxing ${ }^{6,7,10}$ however, a silicone putty guide is bulky and not practical for posterior use and requires additional procedures for the fabrication of interim restorations. The guide made of a thermoplastic sheet is easy to use and translucent, which allows visual evaluation as well as measurement of the clearance underneath the matrix through holes or slots made in the matrix. ${ }^{5,8,9,11}$ An accurate duplication of the axial/occlusal contours is obtained through pressure vacuum and intimate adaptation of the sheet over the duplicate cast of the diagnostic waxing, although the duplication of the occlusal surface details may be compromised. Interim restorations can be fabricated using the same guide at the completion of the tooth preparation procedure; however, evaluation of the reduction may not be accurate because the procedure is performed intraorally, the clear and translucent sheet blends with the tooth color, and a periodontal probe is not calibrated appropriately for fixed prosthodontic procedures.

This article describes a technique for an accurate evaluation of tooth reduction for fixed restorations. The technique demonstrates the fabrication of a color-contrasting positive guide using a silicone occlusal registration material in conjunction with a vacuum-formed clear thermoplastic matrix. The silicone replica of the final restoration is quickly processed within the matrix, separated, and cross-sectioned faciolingually or mesiodistally for visual and quantitative inspections of its thickness outside the mouth using a caliper. Reseating the guide over the prepared tooth permits further intraoral evaluation and identifies areas of adequate-, under-, and over-reduction. The guide is positive, provides color contrast, and is firm for distinct and accurate evaluation of both the occlusal/incisal and axial clearances. This method is simple, accessible, versatile, and can also be used for the tooth modification procedure for the removable partial denture construction. 
A

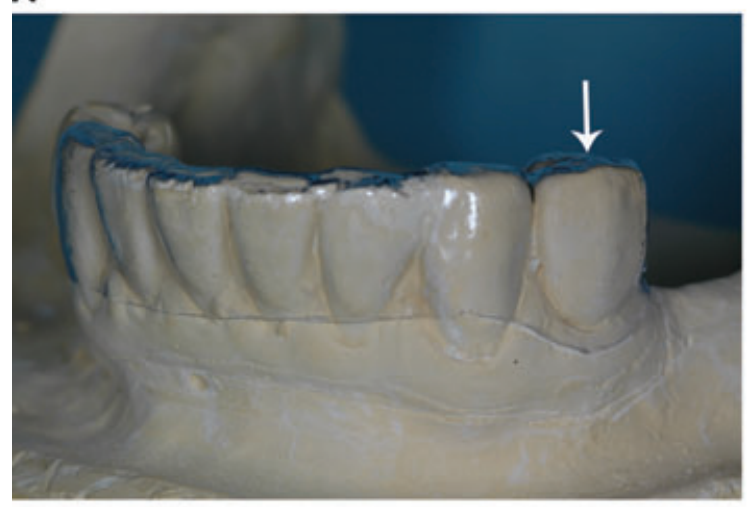

C

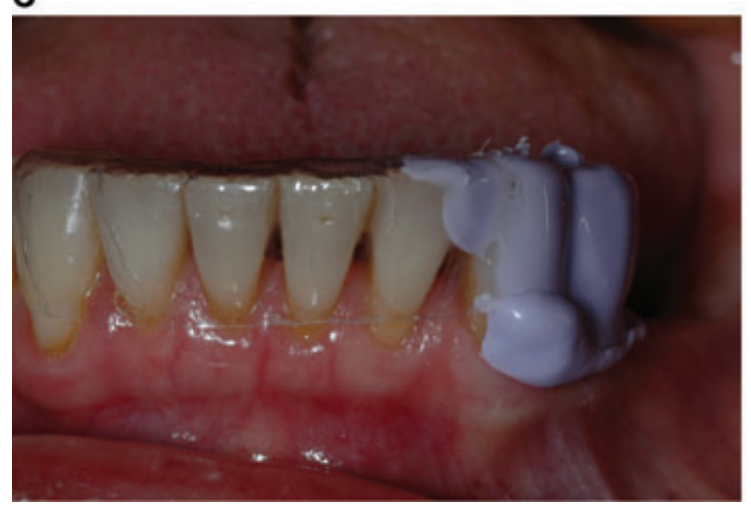

Figure 1 (A) The prepared vacuum-formed clear thermoplastic matrix over the diagnostic waxing duplicate cast. Note arrow indicating an air escape hole. (B) The clear matrix seated intraorally. Note lifting of the matrix resulting from significant alterations of the tooth angulation. (C) The

\section{Technique}

1. Complete the diagnostic waxing to simulate the desired contour and occlusion, then duplicate the wax cast in Type III dental stone (Microstone, Whip Mix Corp, Louisville, $\mathrm{KY})$.

\section{A}

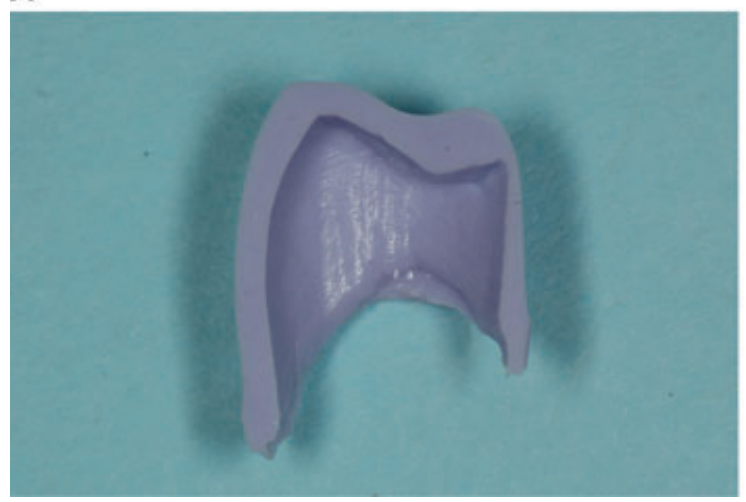

B

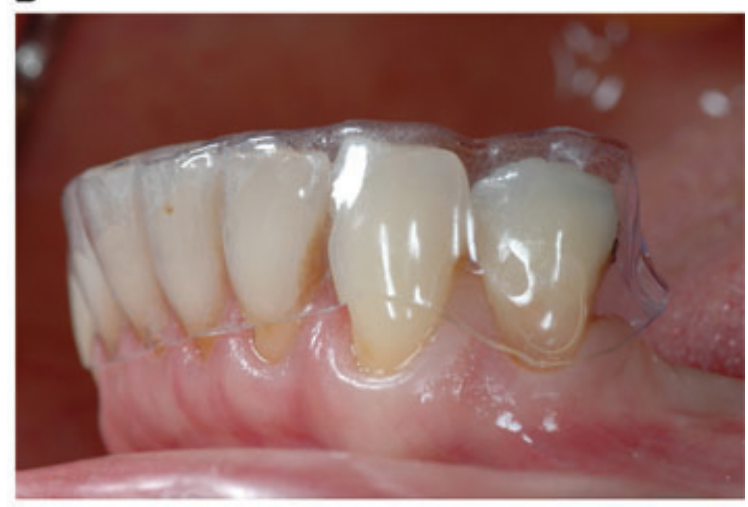

D

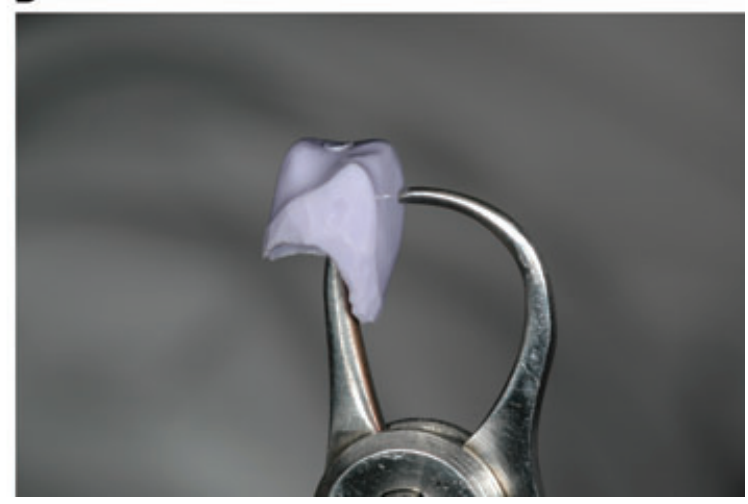

matrix seated intraorally along with a silicone registration material. (D) The processed silicone replica mimicking the contour and thickness of the definitive restoration. Note the precise evaluation and quantification of the thickness of the replica using a caliper.

2. Thermoform a clear plastic matrix (Buffalo Dental MFG, Syesset, NY) over the duplicate cast in the conventional manner. ${ }^{5}$ Separate the vacuum-formed thermoplastic matrix from the cast, trim the excess $1 \mathrm{~mm}$ beyond the gingiva around the prepared tooth using a surgical blade (carbon steel surgical blade number 25, Miltex Inc., York, PA), and

B

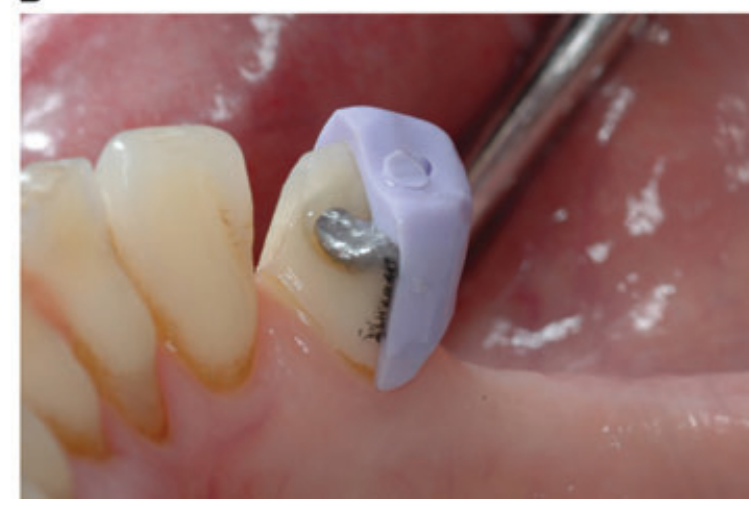

Figure 2 (A) The cross-sectioned silicone replica revealing adequacy/inadequacy of tooth preparation. (B) The cross-sectioned silicone replica seated intraorally identifying the exact area of inadequate tooth reduction. 
make an air escape hole in the center of the prepared tooth of the matrix using a small round bur (Fig 1A).

3. Seat the prepared matrix intraorally to evaluate the tooth angulation and plan the amount of tooth reduction as indicated (Fig 1B). Initiate the tooth preparation and visually evaluate the amount of axial reduction and occlusal clearance with the use of the matrix. To further evaluate the preparation, inject the fast-setting PVS bite registration material (Regisil Rigid, Dentsply Caulk, Milford, DE) into the matrix at the prepared tooth site when the tooth preparation appears to be satisfactory. Seat the matrix intraorally and hold it until the material sets (Fig 1C).

4. Remove the matrix, separate the silicone replica, and trim the excess along the margin of the tooth preparation using scissors. Measure the thickness of the replica using a caliper (Iwanson crown caliper, Miltex Inc.) and determine the adequacy of tooth reduction (Fig 1D).

5. Cross-section the replica faciolingually using a surgical blade (carbon steel surgical blade number 25) (Fig 2A) and reseat the sectioned replica intraorally for further evaluation. Identify the area of inadequate reduction, mark it with a lead pencil (Fig 2B), and modify the tooth preparation as indicated.

6. Proceed to fabricate an interim restoration using the same matrix when the tooth reduction is determined to be adequate and complete.

\section{Summary}

A technique to fabricate a color-contrasting positive tooth reduction guide using a silicone occlusal registration material in conjunction with a vacuum-formed clear thermoplastic matrix has been presented. A clear plastic matrix, thermoformed according to the diagnostic waxing, is used to determine the tooth inclination and evaluate the adequacy of tooth reduction for a fixed restoration. The PVS bite registration material, when processed inside the matrix at the prepared tooth site, will generate a silicone replica of the definitive restoration. The thickness of the replica is measured extraorally using a caliper to quantify the adequacy of the tooth reduction and estimate the thickness of the definitive restoration.

The technical evaluation is facilitated by the color contrast of the replica against the tooth, and precision is facilitated through the use of a rigid replica guide. Initial tooth reduction is carried out with the aid of the clear vacuum-formed thermoplastic matrix. The processed silicone replica is cross-sectioned faciolingually/mesiodistally and positioned on the prepared tooth to determine the accuracy of tooth reduction. When the tooth reduction is complete, an interim restoration is fabricated using the thermoplastic matrix. This method is convenient for all regions of the dentition and applicable to fixed and removable prosthodontic procedures.

\section{References}

1. Rosenstiel SF, Land MF, Fujimoto J: Contemporary Fixed Prosthodontics (ed 4). St. Louis, Elsevier, 2006, pp. 209257

2. Kohal RJ, Gerds T, Strub JR: Effect of different crown contours on periodontal health in dogs. Clinical results. J Dent 2003;31:407-413

3. Wylie SG, Tan HK, Brooke K: Restoring the vertical dimension of mandibular incisors with bonded ceramic restorations. Aust Dent J 2000;45:91-96

4. Douglas RD, Przybylska M: Predicting porcelain thickness required for dental shade matches. J Prosthet Dent 1999;82:143-149

5. Moskowitz ME, Loft GH, Reynolds JM: Using irreversible hydrocolloid to evaluate preparations and fabricate temporary immediate provisional restorations. J Prosthet Dent 1984;51:330-333

6. Fareed K, Solaihim A: Making a fixed restoration contour guide. J Prosthet Dent 1989;61:112-114

7. Gardner LK, Rahn AO, Parr GR, et al: Using a tooth-reduction guide for modifying natural teeth. J Prosthet Dent 1990;63:637-639

8. Tan HK: A preparation guide for modifying the mandibular teeth before making a maxillary single complete denture. J Prosthet Dent 1997;77:321-322

9. Bluche LR, Bluche PF, Morgano SM: Vacuum-formed matrix as a guide for the fabrication of multiple direct patterns for cast posts and cores. J Prosthet Dent 1997;77:326-327

10. Magne P, Douglas WH: Additive contour of porcelain veneers: a key element in enamel preservation, adhesion, and esthetics for aging dentition. J Adhes Dent 1999;1:81-92

11. Livaditis GJ: Indirectly formed matrix for multiple composite core restorations: two clinical treatments illustrating an expanded technique. J Prosthet Dent 2002;88:245-251 